\title{
Short-range correlations studies in collisions of polarized nuclei at Nuclotron-M
}

\author{
V.P. Ladygin ${ }^{1,2, a}$, T. Uesaka ${ }^{3}$, L.S. Azhgirey ${ }^{1}$, Yu.V. Gurchin ${ }^{1}$, A.Yu. Isupov ${ }^{1}$, K. Itoh ${ }^{4}$, M. Janek ${ }^{1,5}$, J.-T. Karachuk ${ }^{1,6}$, \\ T. Kawabata ${ }^{3}$, A.N. Khrenov ${ }^{1}$, A.S. Kiselev ${ }^{1}$, V.A. Kizka ${ }^{1}$, A.B. Kurepin ${ }^{7}$, A.K. Kurilkin ${ }^{1,2}$, P.K. Kurilkin ${ }^{1,2}$, \\ S.M. Piyadin ${ }^{1}$, E.B. Plekhanov ${ }^{1}$, J. Popovichi ${ }^{6}$, S. Rangelov ${ }^{9}$, S.G. Reznikov ${ }^{1}$, P.A. Rukoyatkin ${ }^{1}$, S. Sakaguchi ${ }^{10}$, \\ I.E. Vnukov ${ }^{12}$, and H. Witala ${ }^{13}$ \\ 1 Joint Institute for Nuclear Research, Dubna, Russia \\ 2 Moscow State Institute of Radio-engineering Electronics and Automation, Moscow, Russia \\ 3 Center for Nuclear Study, University of Tokyo, Tokyo, Japan \\ 4 Department of Physics, Saitama University, Urawa,Japan \\ 5 P.J.Šafarik University, Košice, Slovakia \\ 6 Advanced Research Institute for Electrical Engineering, Bucharest, Romania \\ 7 Institute for Nuclear Research, Moscow, Russia \\ 8 Kyushi University, Hakozaki, Japan \\ 9 University of Chemical Technology and Metallurgy, Sofia, Bulgaria \\ 10 RIKEN (the Institute for Physical and Chemical Research), Saitama, Japan \\ 11 University of Tokyo, Tokyo, Japan \\ 12 Belgorod State University, Belgorod, Russia \\ 13 Institute of Physics, Jagiellonian University, Krakow, Poland
} V.A. Krasnov ${ }^{1,7}$, N.B. Ladygina ${ }^{1}$, D. Lipchinski ${ }^{6}$, A.N. Livanov ${ }^{1,7}$, Y. Maeda ${ }^{8}$, A.I. Malakhov ${ }^{1}$, G. Martinska ${ }^{5}$, S. Nedev $^{9}$, H. Sakai ${ }^{3,11}$, Y. Sasamoto ${ }^{10}$, K. Sekiguchi ${ }^{10}$, M.A. Shikhalev ${ }^{1}$, K. Suda ${ }^{10}$, A.A. Terekhin ${ }^{1,12}$, J. Urban ${ }^{5}$, T.A. Vasiliev ${ }^{1,2}$,

\begin{abstract}
The status and prospects of 2-nucleon and 3-nucleon short range correlations (SRCs) studies at Nuclotron-M (JINR) are presented. This program is focused on the investigations of the spin part of SRCs with polarized deuteron beam from new high intensity polarized deuterons ion source which is under development at JINR. The wide experimental program on the systematic studies of the polarization effects in $d p$ - elastic scattering, $d p$ - nonmesonic breakup, $d d \rightarrow{ }^{3} \mathrm{Hen}\left({ }^{3} \mathrm{H} p\right)$ and $d^{3} \mathrm{He} \rightarrow p^{4} \mathrm{He}$ reactions sensitive to SRCs using both internal and extracted beam at Nuclotron-M is presented.
\end{abstract}

\section{Introduction}

Short range correlations (SRCs) of nucleons in nuclei is the subject of intensive theoretical and experimental works during last years. Since SRCs have densities comparable to the density in the center of a nucleon which is about $\rho \sim 5 \rho_{0}\left(\rho_{0} \approx 0.17 \mathrm{fm}^{-3}\right)$, they can be considered as the drops of cold dense nuclear matter [1]. These studies explore a new part of the phase diagram and very essential to understand the evolution of neutron stars.

The results obtained at BNL [2], SLAC [3] and JLAB $[4,5]$ clearly demonstrate that: (i) more than $90 \%$ all nucleons with momenta $k \geq 300 \mathrm{MeV} / c$ belong to $2 \mathrm{~N} \mathrm{SRC}$; (ii) probability for a given proton with momenta $300 \leq k \leq$ $600 \mathrm{MeV} / c$ to belong to $p n$ correlation is $\sim 18$ times larger than for $p p$ correlations; (iii) probability for a nucleon to have momentum $\geq 300 \mathrm{MeV} / c$ in medium nuclei is $\sim 25 \%$; (iv) $3 \mathrm{~N}$ SRC are present in nuclei with a significant probability [6]. However, still many open questions persist and

\footnotetext{
a e-mail: vladygin@jinr.ru
}

further investigations are required both from the experimental and theoretical sides. For instance, the experimental data on the spin structure of $2 \mathrm{~N}(\mathrm{I}=1)$ and $3 \mathrm{~N}$ SRC are almost absent.

The main tools to study SRCs at hadronic facilities can be deuteron structure investigations at large internal momenta allowing to explore $2 \mathrm{~N} \mathrm{SRC}$ with $I=0 ;{ }^{3} \mathrm{He}$ structure to understand the role of $2 \mathrm{~N}$ SRC with $I=1$ and $3 \mathrm{~N}$ SRC; nuclei breakup $A(p, p p) X, A(p, p n) X, A(p, p p p) X$ etc. with the detection of few nucleons in the final state. The greate importance is the study of the spin effects in these reactions because the data on the SRCs spin structure are scarce. Nuclotron-M and NICA will allow to investigate the spin effects for multi-nucleon correlations in a wide energy range.

The model of $2 \mathrm{~N}$ and $3 \mathrm{~N}$ correlations at low and moderate energies (below pion threshold production) can be built from the boson-nucleon picture of strong interaction. During last several years a new generation of nucleonnucleon potentials are built (Nijmegen, CD-Bonn, AV-18 etc.). These potentials reproduced the $\mathrm{NN}$ scattering data 
up to $350 \mathrm{MeV}$ with very good accuracy. But these potentials cannot reproduce triton binding energy (underbinding is $0.8 \mathrm{MeV}$ for CD-Bonn), deuteron-proton elastic scattering and breakup data. Incorporation of three nucleon forces (3NF), when the interaction depends on the quantum numbers of the all three nucleons, allows to reproduce triton binding energy and unpolarized deuteronproton elastic scattering and breakup data (see [7] and references therein). The contribution of $3 \mathrm{NF}$ is found to be up to $30 \%$ in the vicinity of so called "cross section minimum" (Sagara discrepancy) for deuteron-proton elastic scattering at intermediate energies $[8,9]$. However, the use of different 3NF models in Faddeev calculations can not reproduce polarization data intensively accumulated during last decade at different facilities [8]-[14].

On the other hand, $p d$ - elastic scattering cross section data obtained already at $250 \mathrm{MeV}$ [11] cannot be reproduced by the Faddeev calculations with the inclusion of modern $3 \mathrm{NF}$. The authors stated that the reason of this discrepancy can be neglecting by new type of shortrange $3 \mathrm{NF}$. These forces can be built within approaches beyond one-boson-exchange. For instance, in the dressed bag model [15] 3NF comes from the interaction between intermediate six-quark state dressed by $\sigma$-field and the third nucleon. The description of $2 \mathrm{~N}$ and $3 \mathrm{~N}$ correlations at the energies higher than several hundreds $\mathrm{MeV} /$ nucleon should be obtained within QCD [1].

The main goal of the $D S S$-project at Nuclotron-M is the systematic studies of the polarized deuteron-induced reactions at Nuclotron-M. The program includes two main directions.

- The measurements of the cross section, vector $A_{y}$ and tensor $A_{y y}$ and $A_{x x}$ analyzing powers in $d p$ - elastic scattering at large angles in cms in the energy range $0.3-$ $2.0 \mathrm{GeV}$ [16] and in $d p$-non-mesonic breakup at the energies below $500 \mathrm{MeV}$ for different kinematic configurations of two final protons [17] at Internal Target Station (ITS) [18] at Nuclotron-M.

- The measurements of the cross section, tensor analyzing power $T_{20}$ and spin correlation parameter $C_{y, y}$ in the $d^{3} \mathrm{He} \rightarrow p\left(0^{\circ}\right)^{4} \mathrm{He}$ reaction [19]-[21] at the energies between 1000 and $2000 \mathrm{MeV}$ using polarized ${ }^{3} \mathrm{He}$ target [22] and extracted polarized deuteron beam from new polarized ion source [23] at Nuclotron-M.

For these studies the development of the efficient polarimetry for deuteron and nucleon beams at intermediate and high energies at Nuclotron-M is necessary [16, 24, 25].

\section{Measurements at Internal Target Station}

The ITS setup is well suited for study of energy dependence of polarization observables for the deuteron-proton elastic scattering and deuteron breakup reaction with the detection of two protons at large scattering angles [16].

For these purposes the $\mathrm{CH}_{2}$-target of $10 \mathrm{mkm}$ thick is used for the measurements. The yield from carbon content of the $\mathrm{CH}_{2}$-target is estimated in separate measurements using carbon wire. The monitoring of the intensity is done from the detection of $p p$ - quasielastic scattering at $90^{\circ}$ in cms by the scintillation counters placed in the horizontal plane. The detection of the $d p$ - elastic events is done by the coincidence measurements of the proton and deuteron. The detectors are placed in the horizontal plane only for the cross section measurements and in the both horizontal and vertical planes for the analyzing powers measurements. The selection of the $d p$-elastic events is done by the correlation of the energy losses in plastic scintillators for deuteron and proton and their time-of-flight difference. The interaction point for each event is reconstructed by the target position monitor [26].

The measurements of the deuteron analyzing powers in $d p$ - elastic scattering have been performed at ITS using polarized beam from polarized ion source POLARIS [27] at the energies 880 and $2000 \mathrm{MeV}[24,25]$. The use of large amount of the scintillation counters allowed cover wide angular range. The measurement of the beam polarization has been performed at $270 \mathrm{MeV}$ where the data on the tensor and vector analyzing powers based on the absolute calibration of the beam polarization exist [28].

\section{$2.1 d p$-elastic scattering at $880 \mathrm{MeV}$}

The results on the angular dependence of the vector $A_{y}$ and tensor $A_{y y}$ and $A_{x x}$ obtained at $880 \mathrm{MeV}$ are shown in Fig. 1 by the solid symbols. The solid, dashed and dash-dotted lines are the results of the Faddeev calculations [29] using CD-Bonn nucleon-nucleon potential [30], of the relativistic multiple scattering calculations [31] using CD-Bonn [30] deuteron wave function (DWF), and the optical potential calculation [32] with the dibaryon DWF [15], respectively. One can see that Faddeev and relativistic multiple scattering models give good description of the data except for $A_{x x}$.
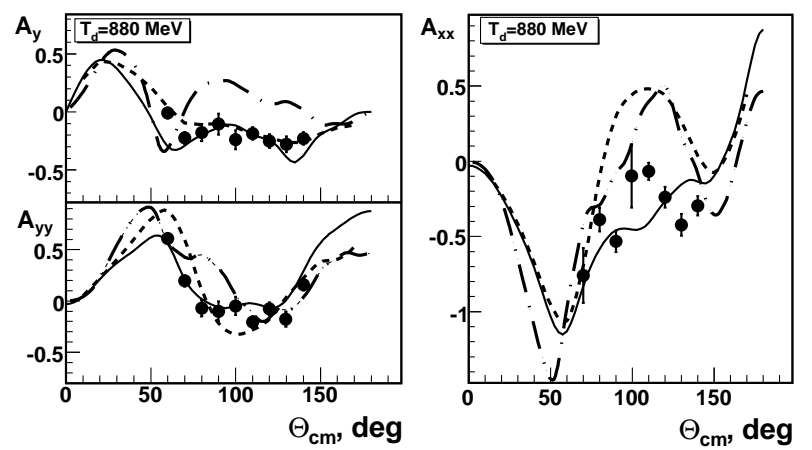

Fig. 1. Vector $A_{y}$, tensor $A_{y y}$ and $A_{x x}$ analyzing powers in $d p$ elastic scattering at $880 \mathrm{MeV}[24,25]$. The lines are the predictions of different models $[29,31,32]$ (explained in the text).

On the other hand, Faddeev calculations [29] fail to reproduce the cross section at the angles larger than $90^{\circ}$, while relativistic multiple scattering calculations [33] give much better agreement with the data at the angles between 
$60^{\circ}$ and $130^{\circ}$. The differential cross section of $d p$ - elastic scattering at the deuteron kinetic energy of $880 \mathrm{MeV}$ is presented in Fig. 2 as a function of the c.m. scattering angle. The full circles and squares are the data from refs.[34] and [35], respectively. The dashed and solid lines are the results of the calculations performed within relativistic multiple scattering model [33] without and with taking into account the rescattering, respectively.

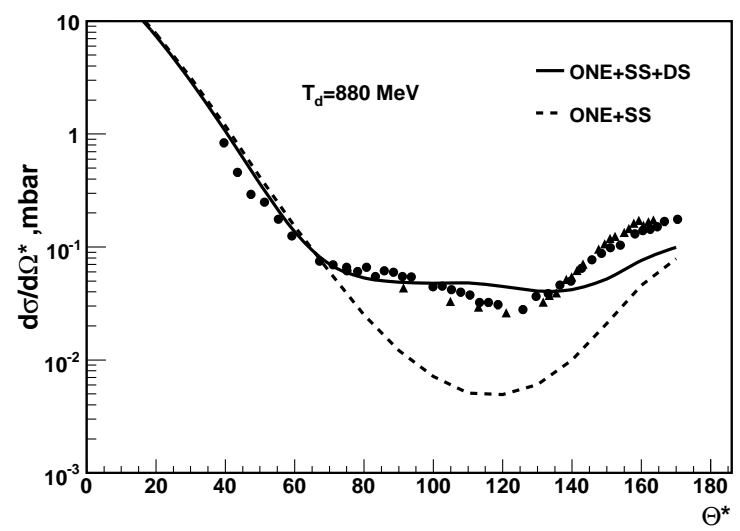

Fig. 2. The differential cross section of $d p$ - elastic scattering at the deuteron kinetic energy of $880 \mathrm{MeV}$ as a function of the c.m. scattering angle. The full circles and squares are the data from refs.[34] and [35], respectively. The dashed and solid lines are the results of the calculations perfomed within relativistic multiple scattering model [33] without and with taking into account the rescattering, respectively.

One can see a reasonable agreement between the calculations [33] and the experimental data [34,35] in the vicinity of the "cross section minimum". One of the reasons of some discrepancy in this region can be 3N SRCs, which are not included in the calculations. These SRCs can be responsible for the deviation between the calculations [31] and data on the analyzing powers $[24,25]$ also.

The shape of the differential cross section for the $d p$ elastic scattering at $500 \mathrm{MeV}$ obtained at ITS at Nuclotron [16] is in good agreement with the RCNP data [11]. It is planned to perform the systematic measurements of the $d p$ - elastic scattering cross section and analyzing powers at the energies 200-500 MeV/nucleon at ITS.

\subsection{Energy dependence of the $d p$ - elastic scattering analyzing powers}

The dependences of the tensor $A_{y y}$ and vector $A_{y}$ analyzing powers in $d p$ - elastic scattering obtained at the fixed angles of $60^{\circ}, 70^{\circ}, 80^{\circ}$ and $90^{\circ}$ in the c.m. as a function of transverse momentum $p_{T}$ are shown in Fig. 3 and in Fig. 4, respectively. The open and solid symbols represent the data obtained at RIKEN, Saclay, ANL [8-10,36-38] and at Nuclotron [24,25], respectively.

The values of $A_{y y}$ are positive at small $p_{T}$ and change the sign at $p_{T} \sim 600-650 \mathrm{MeV} / c$ as in the case of deuteron

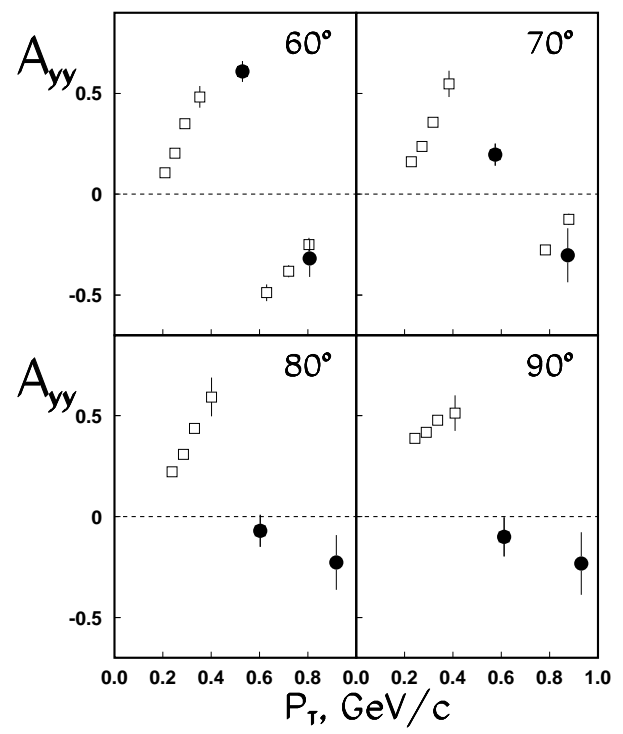

Fig. 3. Tensor $A_{y y}$ analyzing power in $d p$ - elastic scattering obtained at the fixed angles of $60^{\circ}, 70^{\circ}, 80^{\circ}$ and $90^{\circ}$ in the c.m. as a function of transverse momentum $p_{T}$. The open and solid symbols are the data obtained at RIKEN, Saclay, ANL [8-10,36-38] and at Nuclotron [24,25], respectively.

inclusive breakup [39]. The negative sign of $A_{y y}$ is observed at large $p_{T}$. It would be interesting to extend the range of the measurements to larger $p_{T}$, where the manifestation of non-nucleonic degrees of freedom is expected.

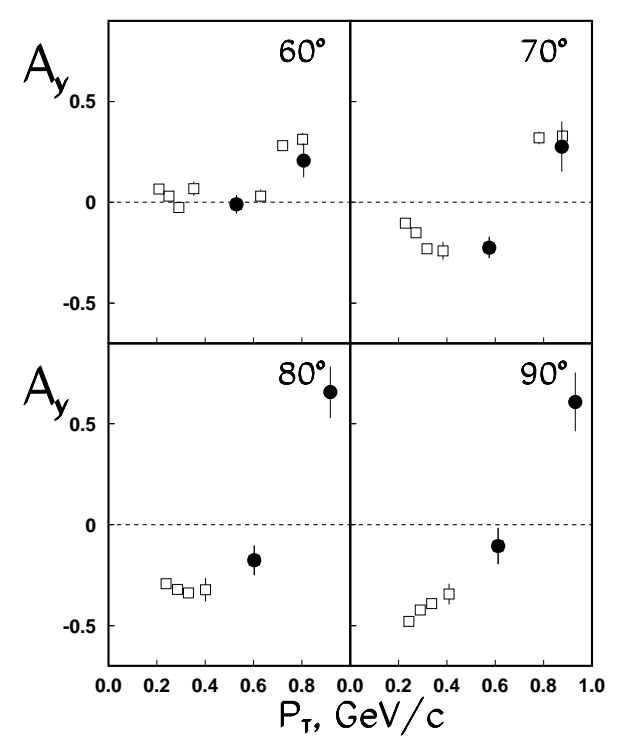

Fig. 4. Vector $A_{y}$ analyzing power in $d p$ - elastic scattering obtained at the fixed angles of $60^{\circ}, 70^{\circ}, 80^{\circ}$ and $90^{\circ}$ in the c.m. as a function of transverse momentum $p_{T}$. The symbols are the same as in Fig. 3. 
The change of the sign is also observed for the vector $A_{y}$ analyzing power values at $p_{T} \sim 600-700 \mathrm{MeV} / c$ at large angles in the c.m. $A_{y}$ has small negative values at low $p_{T}$, but it achieves large positive values at $p_{T}$ higher $\sim 700 \mathrm{MeV} / c$. It should be noted that large positive values of the single spin asymmetry is observed in $p p$ - elastic scattering at high energies and large $p_{T}$ (so called Krisheffect [40]). For $d p$ - elastic scattering such effect is observed at rather low $p_{T}$. Further precise measurements are required to understand the reason of such behavior.

The study of the energy dependence of the $d p$ - elastic scattering analyzing powers at large $p_{T}$ is one of the tools to study spin effects in cold dense matter.

\section{$2.3 d p$ - nonmesonic breakup}

The study of $d p$ - breakup reaction in different kinematic configurations gives an opportunity to select the regions of phase space where the observables are sensitive mostly to $2 \mathrm{NF}$ or $3 \mathrm{NF}$. While the breakup data at the energies of $65-$ $135 \mathrm{MeV} /$ nucleon [41-44] are quite systematic, the lack of the experimental information exists at higher energies. The predictions of the polarization observables and cross section for $d p$ - non-mesonic breakup at $400 \mathrm{MeV}$ for different kinematic configurations of the final protons are given in ref. $[45,46]$. The observables for some kinematic configurations demonstrate strong sensitivity to the contribution of $3 \mathrm{NF}$.

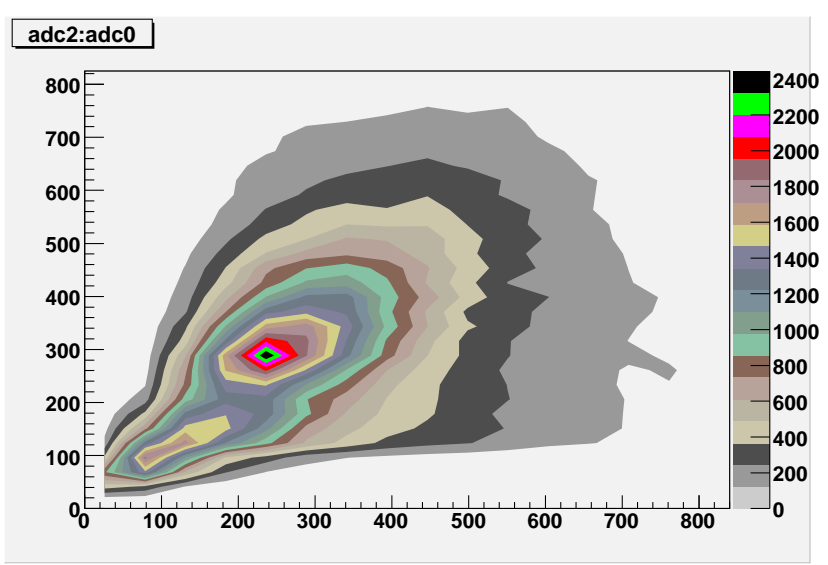

Fig. 5. The correlation of the energy depositions in two $E$ counters obtained at ITS with $2.3 \mathrm{GeV}$ deuteron beam [17].

The $d p$ - non-mesonic breakup reaction will be investigated at ITS at Nuclotron using $\triangle E-E$ techniques for the detection of two final protons. Monte-Carlo simulation shown the feasibility of such techniques for the energies $T_{d} \leq 500 \mathrm{MeV}$ [47]. Each detector consists of two scintillation counters: the first and the second ones are with $1 \mathrm{~cm}$ and $20 \mathrm{~cm}$ scintillators in length, respectively. The diameter of the $E$-counter scintillator is $10 \mathrm{~cm}$. The use 8-10 $\Delta E-E$ detectors simultaneously will allow to measure the observables for many kinematic configurations. The results on the amplitudes signals correlation for two
$E$ detectors obtained during beam test run in June 2008 at ITS using $2.3 \mathrm{GeV}$ deuterons [17] are presented in Fig. 5. The measurements will be performed at the energies below $250 \mathrm{MeV} /$ nucleon at the same time with the measurements of $d p$ - elastic scattering. However, additional time will be required to estimate reliably contribution from the carbon content of $\mathrm{CH}_{2}$ target.

The measurements of the cross sections of $d p$ - elastic scattering and $d p$ - nonmesonic breakup can be done with the current unpolarized ion source. The realization of the spin program at ITS can be started with the intensity of the POLARIS $\left(\sim 2 \cdot 10^{8} \mathrm{ppp}\right)$ [27] maintained from the present value by the recovering of the intensity losses during the injection into Nuclotron ring. The energy scan of the $d p$ elastic scattering observables can be done with new high intensity PIS [23].

\section{Measurements at extracted beam}

The goal of the ${ }^{3} \mathrm{He}(d, p)^{4} \mathrm{He}$ reaction study at Nuclotron$\mathrm{M}$ is to understand the reasons of the long staying puzzle, namely, the behavior of the tensor analyzing power $T_{20}$ in $d p$ - backward elastic scattering $[48,49]$. While $t_{20}$ data in $e d$ - elastic scattering obtained at JLAB [50] and $T_{20}$ data in $d p$ - inclusive breakup $[51,52]$ can be explained by using the conventional deuteron structure functions and additional to the Born approximation mechanisms, the $T_{20}$ in $d p$ - backward elastic scattering demonstrate unexplained strange structure at the internal momentum $k \sim 0.3-0.5$ $\mathrm{GeV} / c$ in the vicinity of the $D$ - wave dominance in the deuteron.

The experiments performed at RIKEN at the energies below $270 \mathrm{MeV}$ have shown that the polarization correlation coefficient

$$
C_{/ /}=1-\frac{1}{2 \sqrt{2}} T_{20}+\frac{3}{2} C_{y, y}
$$

for the ${ }^{3} \mathrm{He}(d, p){ }^{4} \mathrm{He}$ reaction may be a unique probe to the D-state admixture in deuteron [19]. The usefulness of this observable to investigate the D-state admixture is attributed to the strong spin-selectivity in neutron capture process by ${ }^{3} \mathrm{He}$ nucleus, i.e., spins of transferred neutron and ${ }^{3} \mathrm{He}$ must be anti-parallel to each other in order to form ${ }^{4} \mathrm{He}$ in the final state. In the Born approximation, the expression for $C_{/ /}$is proportional to the D-state fraction in deuteron as

$$
C_{/ /}=\frac{9}{4} \frac{w^{2}}{u^{2}+w^{2}}
$$

where $u$ and $w$ are the S- and D-state wave functions of deuteron in momentum space. This is a marked contrast to $T_{20}$ and $\kappa_{0}$ for $d p$ backward elastic scattering which include $\mathrm{S}$ - and D-state interference term ( $u w$-term) together with a $w^{2}$-term. It is thus expected that $C_{/ /}$may be a candidate to provide an information on the deuteron structure complementary to those from $T_{20}$ and $\kappa_{0}$ obtained in $d p$ backward elastic scattering [48]. 


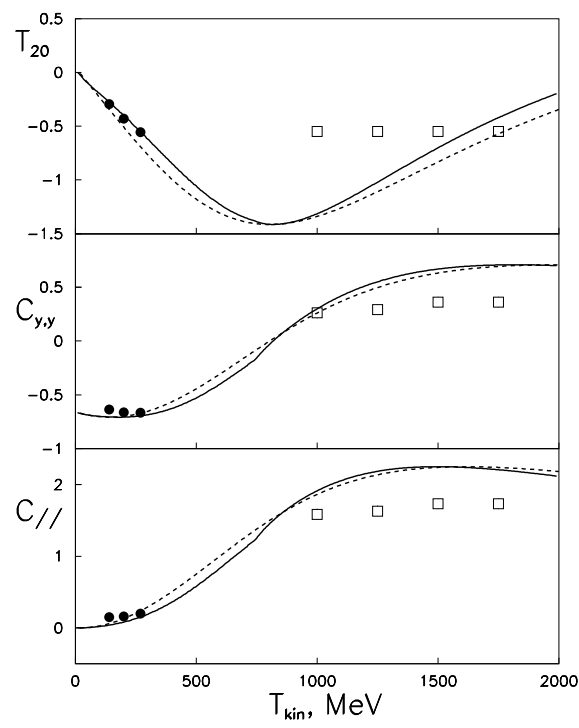

Fig. 6. Tensor analyzing power $T_{20}$, spin correlation $C_{y, y}$ and polarization correlation coefficient $C_{/ /}$for the ${ }^{3} \mathrm{He}(d, p)^{4} \mathrm{He}$ reaction. The dashed and solid lines represent one-nucleon exchange calculations without [20] and taking into account the Fermi motion in the target nucleus, respectively. The full symbols are the data obtained at RIKEN [21]. The open squares show the expected precision for the data at Nuclotron-M.

Tensor analyzing power $T_{20}$, spin correlation $C_{y, y}$ and polarization correlation coefficient $C_{/ /}$for the ${ }^{3} \mathrm{He}(d, p){ }^{4} \mathrm{He}$ reaction are shown in Fig. 6. Solid lines in the figures represent calculations based on an impulse approximation proposed in Ref. [20]. In the calculation, the Fermi motion in the target nucleus is taken into account [21]. The full symbols are the data obtained at RIKEN [21]. The open squares show the expected precision for the data at Nuclotron-M.

The main goal of the experiment is to obtain the data on $C_{/ /}$in the energy region of $1.0-1.75 \mathrm{GeV}$, where the contribution from the deuteron D-state is expected to reach a maximum in one-nucleon exchange approximation, to obtain new information on the strange structure observed in the behavior of $T_{20}$ in the $d p$-backward elastic scattering and to realize experiment on the full determination of the matrix element of the ${ }^{3} \mathrm{He}(d, p)^{4} \mathrm{He}$ reaction in the model independent way. These data will help us also to understand the short-range spin structure of deuteron and effects of non-nucleonic degrees of freedom. For these purposes polarized deuteron beam from new PIS [23] and polarized ${ }^{3} \mathrm{He}$ target developed at CNS of Tokyo University [22] and modified for present experiment at Nuclotron-M will be used.

The data on the analyzing powers in $d\left(d,{ }^{3} \mathrm{He}\right) n$ and $d\left(d,{ }^{3} \mathrm{H}\right) p$ obtained at 270 and $200 \mathrm{MeV}$ [53] are shown in Fig. 7. The curves are the calculation within onenucleon-exchange approximation using standard deuteron and ${ }^{3} \mathrm{He}\left({ }^{3} \mathrm{H}\right)$ wave functions [54]. The data demonstrate the sensitivity to the $D / S$ waves ratio in these light nu- clei at short internucleonic distances. However, the calculations [54] fail to reproduce the data. The reason of the discrepancy can be in the non-adequateness of the $3 \mathrm{~N}$ bound state spin structure and/or more complicated reaction mechanism. The multiple scattering calculations are in progress now [33].
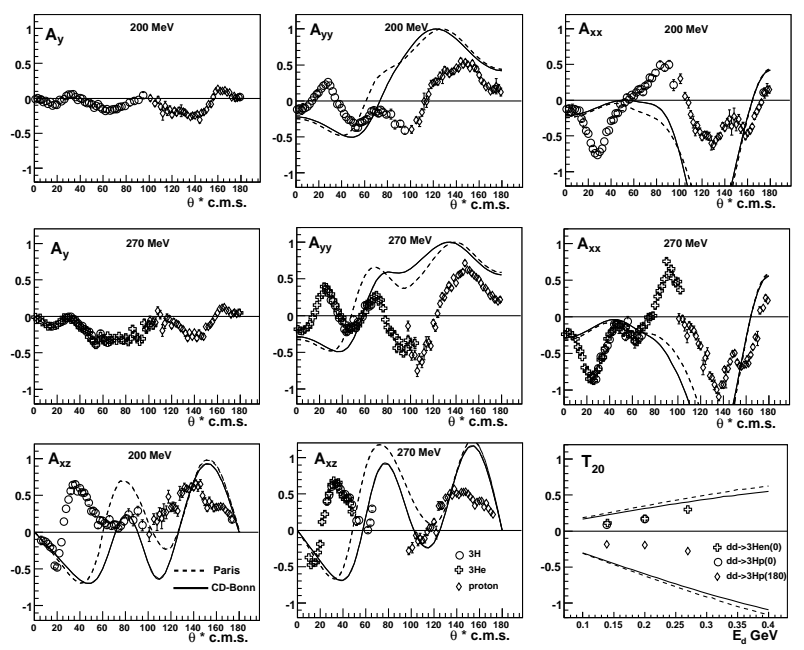

Fig. 7. The data on the analyzing powers in $d\left(d,{ }^{3} \mathrm{He}\right) n$ and $d\left(d,{ }^{3} \mathrm{H}\right) p$ at 270 and $200 \mathrm{MeV}$ [53]. The curves are the calculation within one-nucleon-exchange approximation [33].

The measurement of the tensor analyzing power $T_{20}$ in the $d(d, p)^{3} \mathrm{H}$ and $d\left(d,{ }^{3} \mathrm{He}\right) n$ reactions at the deuteron energies $1-2 \mathrm{GeV}$ can be performed using the same experimental setup as for the ${ }^{3} \mathrm{He}(d, p){ }^{4} \mathrm{He}$ reaction studies.

\subsection{Polarized ${ }^{3} \mathrm{He}$ target}

Several modifications to the existing CNS spin-exchangetype polarized ${ }^{3} \mathrm{He}$ target [22] have been introduced for experiment at Nuclotron-M.

A Fiber Array Packaged Laser(COHERENT FAP-7930C-800-LB) was installed to improve the optical pumping efficiency. Small light emittance due to the introduction of the fiber provides large transmission efficiency through optical elements and good parallelness of the irradiated beam on the target cell.

Correction coils to control the magnetic field homogenity has been introduced. The coils are placed inside of larger Helmholtz coils which generate a homogeneous holding field of $\sim 15 \mathrm{G}$. The purpose is to extend the area of the field homogeneity better than $10^{-3} \mathrm{~cm}^{-1}$ to cover the enlarged target cell size of $30 \mathrm{~cm}$.

A diagnostics system to measure the ${ }^{3} \mathrm{He}$ density and polarization accurately has established. Target density measurement with the pressure broadening method for the $\mathrm{Rb} D_{1}$ was introduced. By using the optical method, one can deduce the target density with high precision and high accuracy (relative error of $\sim 2 \%$ ). New systems for the measurements of the polarization of the target using NRM 
and density of the target has been manufactured, installed and tested.

The modified target is ready for the experiment at Nuclotron-M and has been used in the experiment on the polarization observables in the ${ }^{3} \mathrm{He}(p, p) p X$ reaction studies at RCNP [55].

\subsection{Polarized deuteron beam}

To study the spin observables for the ${ }^{3} \mathrm{He}(d, p){ }^{4} \mathrm{He}$ reaction new high intensity PIS [23] with the full intensity of $\sim 2 \cdot 10^{10} \mathrm{ppp}$ is required. In this case one can measure $T_{20}$ and $C_{y, y}$ simultaneously using polarized ${ }^{3} \mathrm{He}$ target of $30 \mathrm{~cm}$ thick. New source will allow to have wide number of spin states with different combination of vector and tensor polarizations. In the experiment it is planned to use the modes with the following ideal values of $\left(p_{z}, p_{z z}\right)$ : $(0,0),(0,-2),(2 / 3,0)$ and $(-1 / 3,+1)$. The polarization of the beam will be measured by the ITS polarimeter based on the asymmetry measurement of $d p$ - elastic scattering [24, 25].

\subsection{Beam parameters and setup layout}

The position of the polarized ${ }^{3} \mathrm{He}$ target will be $\sim 8 \mathrm{~m}$ downstream of the focus F3 of the Nuclotron extraction line. The results of the deuteron beam parameters measurements performed at the energies between 1000 and 2000 $\mathrm{MeV}$ at the focus F3 of the extraction line at Nuclotron [56] demonstrate that the beam parameters from Nuclotron are good enough to perform the experiment. However, it is necessary to modify the beam line to remove additional matter along the extraction line to reduce the multiple scattering of the initial beam.

The detection system will be placed downstream focus F5 of the VP1 beam line. Calculations for new magnetic optics have been performed [57]. The emittance of the primary beam assumed is $10-20 \pi \mathrm{mm} \cdot \mathrm{mr}$, the size of the beam $(2 \sigma)$ at the target is about $0.5 \mathrm{~cm}$ both in the $X$ and $Y$ direction. The fields in the magnetic elements correspond to the momentum of the proton from the reaction ${ }^{3} \mathrm{He}(d, p){ }^{4} \mathrm{He}$. The primary deuteron beam and secondaries are separated by the magnet with the bending angle of $\sim 140 \mathrm{mr}$ placed upstream focus F4. Optical calculations show that the size of the secondary beam in the focus F5 is $2-3 \mathrm{~cm}$. The momentum and angular acceptances are $\Delta p / p= \pm 4 \%$ and $\sim 3$ msr $\left(4 \sigma_{\theta_{x}} \sigma_{\theta_{y}}\right)$, respectively. The angular acceptance can be increased in approximately 2-3 times by the use of the lenses with larger diameter downstream the target.

In the experiment it will be necessary to select protons against background deuterons with the same momentum, on the one hand, and to provide a good momentum resolution to select protons from the ${ }^{3} \mathrm{He}(d, p)^{4} \mathrm{He}$ reaction, on the other hand.

The setup will consist of analyzing magnet, scintillation counters and drift chambers. The scintillation counter and a set of drift chambers is placed at the focus F5 upstream the analyzing magnet. The second set of the drift chambers, trigger counters and scintillation counters hodoscope are placed downstream the magnet. The size of the drift chambers placed in front of the analyzing magnet can be about $10 \times 10 \mathrm{~cm}^{2}$ according the calculations for magnetic optics [57].

The identification of the particles will be done by the measurements of their time-of-flight (TOF) over a baseline of about $30 \mathrm{~m}$. Three scintillation counters viewed from both sides by XP2020 will be used for the trigger purpose and as the start detectors for the time-of-flight measurements. The additional set of the scintillation counters are placed in the focus F4 to provide time-of-flight measurements. The time-of-flight baseline of about $30 \mathrm{~m}$ is enough to separate the deuterons and protons with the same momentum up to $3.0 \mathrm{GeV} / \mathrm{c}$.

The momentum of the particles is reconstructed via the measurements of the bending angle in the analyzing magnet. The sets of the drift chambers will be used as the tracking detectors. With the bending angle of $\sim 200 \mathrm{mr}$ the momentum resolution of $\sim 0.1 \%$ will be achieved.

Fast VME based DAQ system will be used for the data taking. However, in the case of large yield of deuterons this background can be suppressed partly by the second level trigger based on the measurements of the particles time-offlight. The preparation of the time-of-flight counters [58], DAQ and HV systems [17] is in progress.

\section{Polarimetry at Nuclotron-M}

The spin part of $D S S$ - project requires good knowledge of the deuteron beam polarization and its permanent monitoring during data taking. It crucial also to establish a polarimetry suited for a vector-tensor mixed polarized beam provided by new LHEP PIS [23].

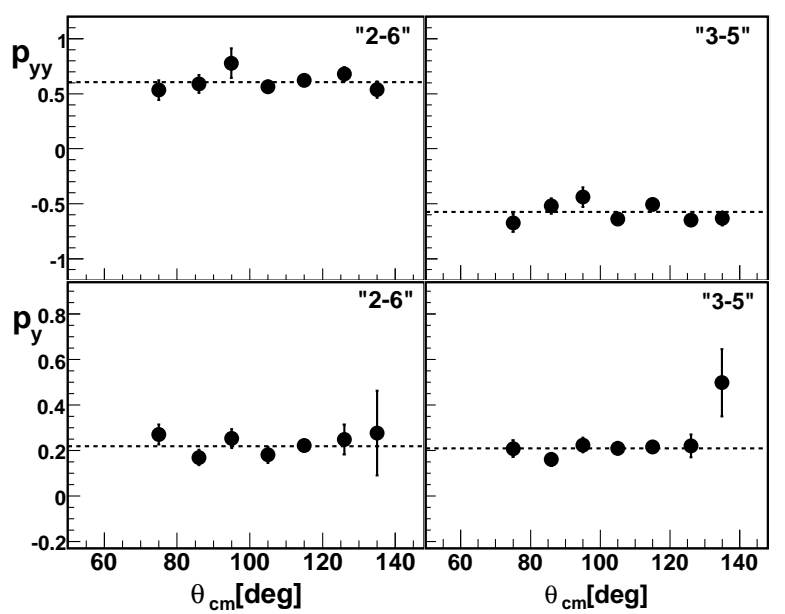

Fig. 8. Results of the beam polarization measurements for different spin modes of POLARIS [27] by ITS polarimeter at 270 $\mathrm{MeV}$.

In the framework of $D S S$ - project new polarimetry scheme at Nuclotron-M will be established. The basic po- 


\section{$19^{\text {th }}$ International IUPAP Conference on Few-Body Problems in Physics}

larimeter in this scheme is the polarimeter at ITS with the $d p$ - elastic scattering at backward angles $\left(\theta_{\mathrm{cm}}>60^{\circ}\right)$ at the energies $270-2000 \mathrm{MeV}$ as the analyzing reaction [16]. This polarimeter has several advantages. Firstly, both vector and tensor analyzing powers for the reaction can have large values. Therefore, such polarimeter can allow to measure both vector and tensor beam polarizations and also the spin direction. Secondly, a kinematic coincidence measurement of deuteron and proton with simple plastic scintillation counters suffices for event identification. This is mainly due to a small background event rate, compared with the forward angles. The use of this reaction as a deuteron polarimetry at $140-270 \mathrm{MeV}$ at large angles has been established at RIKEN [8-10].

The first calibration data obtained at $880 \mathrm{MeV}$ and $2000 \mathrm{MeV}$ demonstrated the feasibility of such polarimeter [59]. The use of large amount of the scintillation counters allowed to cover wide angular range. The results on the vector and tensor beam polarizations for different spin modes of POLARIS [27] by ITS polarimeter at $270 \mathrm{MeV}$ at different angles in c.m. are shown in Fig. 8. The beam polarization values were evaluated from the $d p$ - elastic scattering asymmetries and values of the analyzing powers obtained with high precision from absolute calibration of the beam polarization at RIKEN [28]. The tensor and vector beam polarization values can be obtained with the precision better than $2 \%$.

The ITS polarimeter will be calibrated in a wide energy range with reasonable energy step. The expected precision of the analyzing powers calibration is $2-3 \%$ at the energies below $900 \mathrm{MeV}$. The results on the analyzing powers $A_{y}, A_{y y}$ and $A_{x x}$ in $d p$ - elastic $\left(\mathrm{H}_{2}\right)$ and quasi-elastic $\left(\mathrm{CH}_{2}\right)$ scattering at $880 \mathrm{MeV}$ presented in Fig. 9 [59] demonstrate minor influence of carbon in this energy domain.

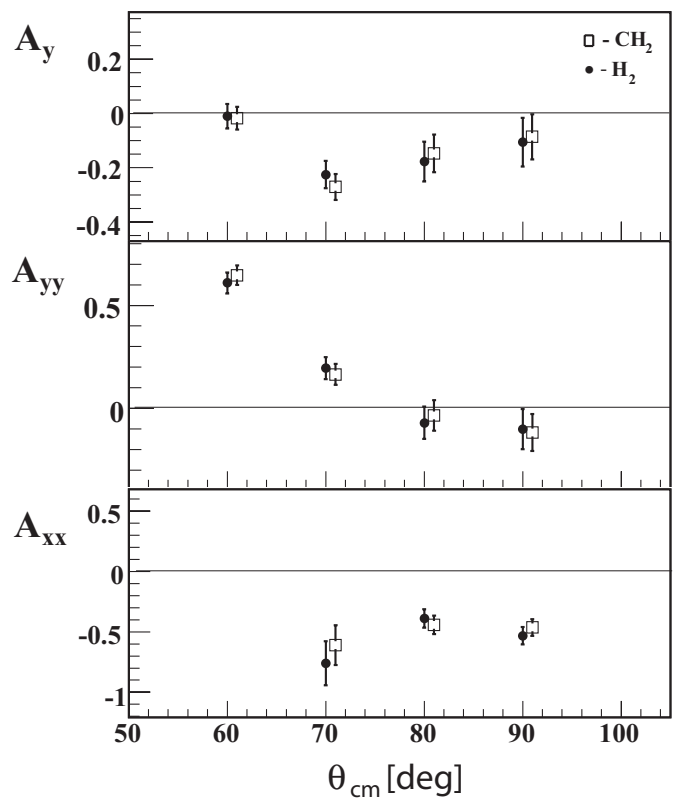

Fig. 9. Analyzing powers $A_{y}, A_{y y}$ and $A_{x x}$ in $d p$ - elastic $\left(\mathrm{H}_{2}\right)$ and quasi-elastic $\left(\mathrm{CH}_{2}\right)$ scattering at $880 \mathrm{MeV}$ [59].
At higher energies (above $1000 \mathrm{MeV}$ ) the carbon content of $\mathrm{CH}_{2}$ gives much higher contribution. However, the results on the behavior of the vector $A_{y}$ and tensor $A_{y y}$ analyzing powers for of $d p$ - quasi-elastic scattering obtained at $2000 \mathrm{MeV}$ [25] demonstrate the sizable values of these analyzing powers. This can simplify significantly the deuteron beam polarimetry at high energies. The expected precision of the analyzing powers calibration is $3-5 \%$ at the energies between 1000 and $2000 \mathrm{MeV}$.

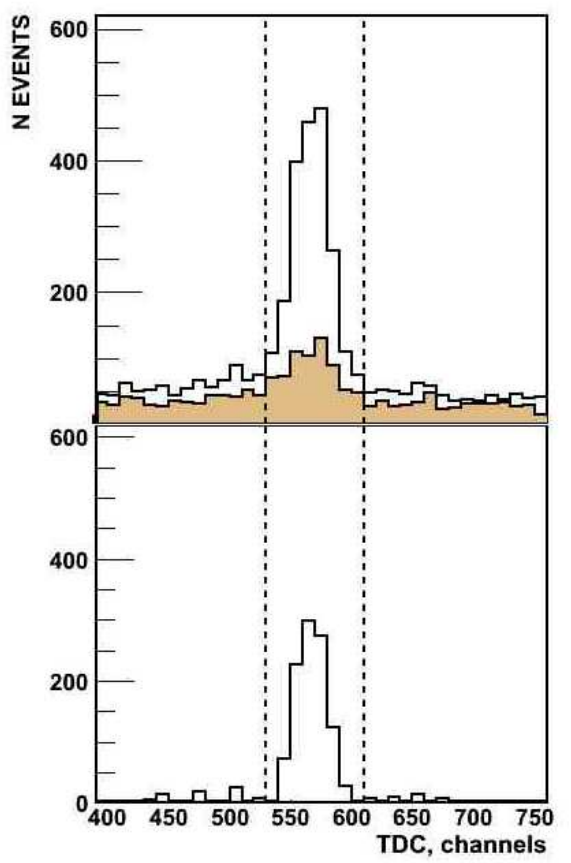

Fig. 10. Selection of the $d p$ - elastic scattering events at $\theta_{l a b}^{d}=8^{\circ}$ and $T_{d}=2000 \mathrm{MeV}$ at extracted Nuclotron beam. The open and shadowed distributions in the upper panel are the events obtained on $\mathrm{CH}_{2}$ and carbon targets, respactively. The results of $\mathrm{CH}_{2}-\mathrm{C}$ subtraction are presented in the bottom panel. The dashed lines are the prompt timing windows for the final selection of the $d p$ elastic events.

Three different polarimeters at external Nuclotron$\mathrm{M}$ beam will be calibrated with the precision 3-5\% at $1600 \mathrm{MeV}$. The vector polarimeter based on quasi-elastic $p p$ - scattering [60] and located in focus F3 is necessary for the program with polarized nucleons. Deuteron inclusive breakup polarimeter [61] with the detection of protons with the momentum $p_{p}=2 / 3 \cdot p_{d}$ can be useful for the experiments where only tensor effects are studied. The use of these both polarimeters simultaneously allowed to measure both tensor and vector polarizations of the extracted beam [39]. Such tensor polarimeter will be based on the use of the magnetic spectrometer for the ${ }^{3} \mathrm{He}(d, p)^{4} \mathrm{He}$ reaction measurements.

New high energy polarimeter based on $d p$ - elastic scattering at forward angles, where both $A_{y}$ and $A_{y y}$ have large values $[37,38]$, will be also located in focus F3. The $d p$ - elastic scattering events will be detected by the 
kinematic coincidence of deuteron and proton with plastic scintillation counters. The final selection of useful events will be performed using amplitude and timing information. The result of the $d p$ - elastic scattering events selection at $\theta_{l a b}^{d}=8^{\circ}$ and $T_{d}=2000 \mathrm{MeV}$ via $\mathrm{CH}_{2}-\mathrm{C}$ subtraction of timing spectra [62] is shown in Fig. 10. The open and shadowed distributions presented in the upper panel in Fig. 10 are the events obtained on $\mathrm{CH}_{2}$ and carbon targets, respactively, while the results of $\mathrm{CH}_{2}-\mathrm{C}$ subtraction are presented in the bottom panel. The dashed lines are the prompt timing windows for the final selection of the $d p$ elastic events.

The goal of the $D S S$ - project is to establish the polarization standard for high energy deuteron beam polarimetry at Nuclotron-M (initially based on the high accuracy $d p$ - elastic scattering data obtained at RIKEN [28]).

\section{Study of SRCs at NICA}

New heavy ion and polarized particles collider NICA is planned for the energies $\sqrt{s_{N N}} \sim 4-12 \mathrm{GeV}$ and up to $\sqrt{s} \sim 27 \mathrm{GeV}$ for $d d$ - and $p p$ - collisions, respectively. The serious advantage of this facility is the availability of polarized deuterons (neutrons). The main topics of the spin studies at NICA is the spin content of nucleon, nuclear and color transparency in spin observables, polarization effects in hyperon production, single and double asymmetries in meson production, $N N$ and light nuclei short-range spin structure [63].

Deuteron and ${ }^{3} \mathrm{He}\left({ }^{3} \mathrm{H}\right)$ spin structure can be studied using two-arms magnetic spectrometer from $d d \rightarrow p X$ [39], $d d \rightarrow{ }^{3} \mathrm{Hen}\left({ }^{3} \mathrm{H} p\right)$ [53], ${ }^{3} \mathrm{He}^{3} \mathrm{He} \rightarrow p p X$ and other reactions. The deuteron internal structure in the $d d \rightarrow p X$ process can be probed at NICA energies up to $p_{T} \sim 2-3$ $\mathrm{GeV} / c$, where different models predict significantly different behavior of the cross section [64]. The results of the cross section data in the $d p \rightarrow p X$ reaction calculations for laboratory frame at $P_{d}=40 \mathrm{GeV} / c$ [64] using standard [30] and covariant DWF [65] for different proton emission angles are shown in Fig. 11 and Fig. 12, respectively. For collider mode such calculations correspond to $\sqrt{s} \sim 5.3$ $\mathrm{GeV} / c^{2}$ and proton emission angles around $90^{\circ}$ with respect to the beam direction. The results strongly deviate for the calculations based on the use of the standard and covariant DWFs [64] as well as from the constituent counting rule prediction $[66,67]$. The cross section data for deuteron breakup reaction at these energies are sensitive also to the problem of hidden color in nuclei via the measurement of the cross section ratio for $N\left(d, p \pi^{+}\right) X$ and $N(d, p) X$ processes [66].

NICA will provide the opportunity to measure tensor analyzing power $A_{y y}$ and polarization transfer coefficient $K_{y}^{y}$ in deuteron inclusive breakup with the proton emission at large $p_{T}$. The data on the tensor analyzing power $A_{y y}$ obtained in the $A(d, p) X$ reaction at different values of $x_{F} \sim 0.61, \sim 0.67, \sim 0.72$ and $\sim 0.78$ and plotted as a function of the proton transverse momentum $p_{T}$ are shown in Fig. 13 a), b), c) and d), respectively. The figure

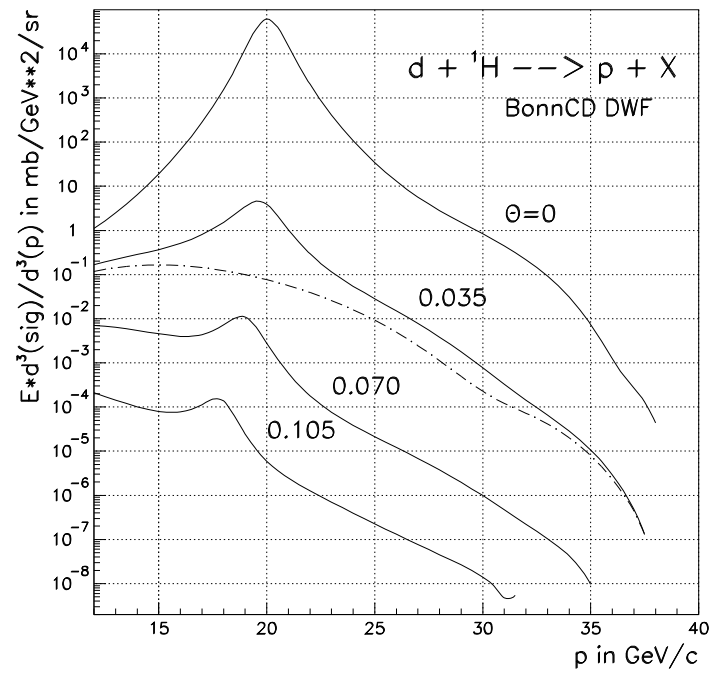

Fig. 11. The calculations for the $d p \rightarrow p X$ reaction cross section at $P_{d}=40 \mathrm{GeV} / c\left(\sqrt{s} \sim 5.3 \mathrm{GeV} / c^{2}\right)$ for different proton emission angles [64] in $m r$ using CD-Bonn DWF [30].

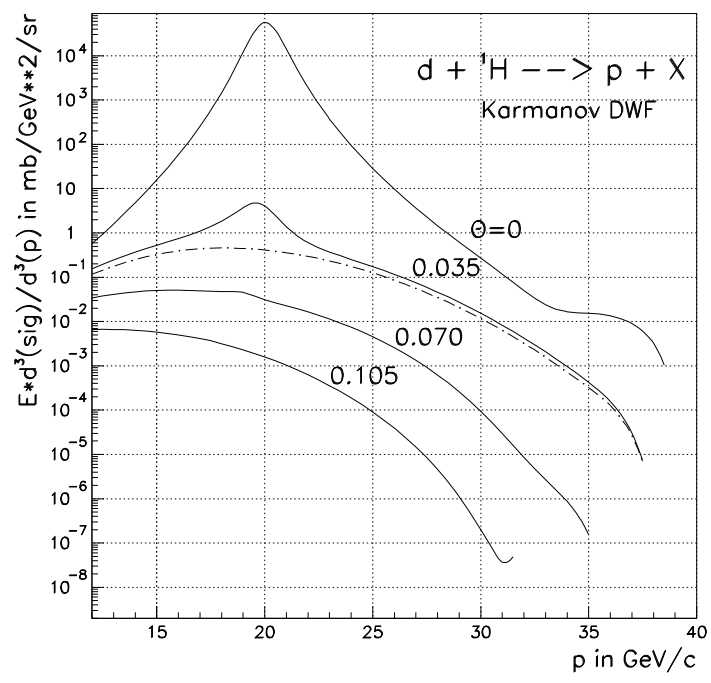

Fig. 12. The calculations for the $d p \rightarrow p X$ reaction cross section at $P_{d}=40 \mathrm{GeV} / c\left(\sqrt{s} \sim 5.3 \mathrm{GeV} / c^{2}\right)$ for different proton emission angles in $m r$ [64] using covariant DWF [65].

is taken from ref.[39]. It is seen that the $A_{y y}$ data for different $x_{F}$ are strongly dependent of the transverse momentum of the protons, $p_{T}$. Values of $A_{y y}$ are positive at small $p_{T}$ and monotonously decrease while transverse momentum increasing for all $x_{F}$ values. On the other hand, $A_{y y}$ values change the sign at $p_{T} \sim 600 \mathrm{MeV} / c$ independently on $x_{F}$ and demonstrate kind of negative asymptotic at large $p_{T}$. Note, that the same features are observed in the behavior of $A_{y y}$ in $d p$ - elastic scattering at large $p_{T}$ (see Fig. 3). 


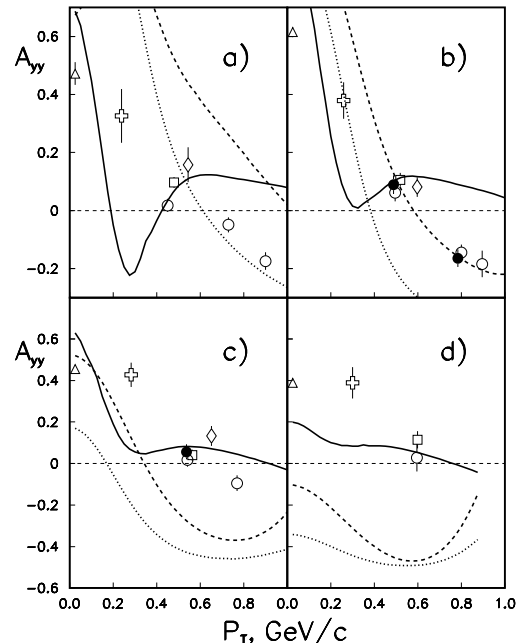

Fig. 13. $A_{y y}$ data for deuteron inclusive breakup plotted versus proton transverse momentum $p_{T}$ at four different fixed values of $x_{F} \sim 0.61,0.67,0.72$ and 0.78, are shown in a), b), c) and d) panels, respectively [39]. The dashed, dash-dotted and solid curves are the results of the calculations using standard $[30,68]$ and covariant [65] DWFs, respectively.

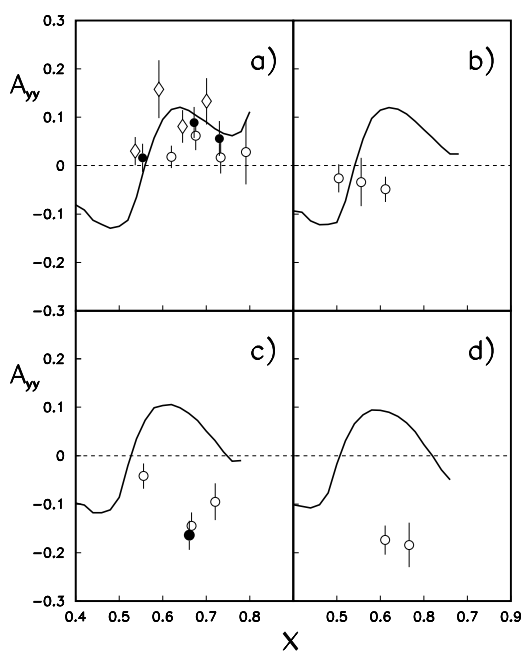

Fig. 14. $A_{y y}$ data plotted as a function of longitudinal momentum fraction $x_{F}$ obtained at fixed $p_{T}$ values of $\sim 550 \mathrm{MeV} / \mathrm{c}, \sim 700$ $\mathrm{MeV} / \mathrm{c}, \sim 800 \mathrm{MeV} / \mathrm{c}$ and $\sim 900 \mathrm{MeV} / \mathrm{c}$ and shown in a), b), c) and d) panels, respectively [39]. The curves are the results of calculation using covariant DWF [65].

In Fig. 14 the $A_{y y}$ data are plotted at different values of transverse momenta $p_{T}$ as a function of $x_{F}$. The data shown in panels a), b), c) and d) correspond to the averaged values of $p_{T} \sim 550 \mathrm{MeV} / \mathrm{c}, \sim 700 \mathrm{MeV} / \mathrm{c}, \sim 800 \mathrm{MeV} / \mathrm{c}$ and $\sim 900$ $\mathrm{MeV} / \mathrm{c}$, respectively. The figure is taken from ref.[39]. The solid curves are the results of the calculations by using covariant DWF [65]. One can see that the $A_{y y}$ data for different values of $p_{T}$ demonstrate a weak dependence on $x_{F}$.
The data obtained at $p_{T} \sim 550 \mathrm{MeV} / \mathrm{c}$ are in a good agreement with the calculations by using covariant DWF [65]. At higher $p_{T} A_{y y}$ data have negative values, while the theory predicts a positive sign in the range of measurement

Therefore, the $A_{y y}$ data clearly demonstrate the dependence on two internal variables, $x_{F}$ and $p_{T}$ (or their combinations). However, the use of the deuteron structure function that depends on two variables [65] does not allow to describe the data. The measurements of the tensor analyzing power $A_{y y}$ and polarization transfer coefficient $K_{y}^{y}$ in deuteron inclusive breakup, $d d \rightarrow p X$, at $p_{T} \geq 1 \mathrm{GeV} / c$ at NICA is feasible and can bring new information on the spin dependence of $2 \mathrm{~N}$ SRCs.

The collider mode gives very serious advantage to study $2 \mathrm{~N}$ and $3 \mathrm{~N}$ SRCs in nuclei from the $A(p, p p) X$ and $A(p, p n) X$ processes because of large coverage of the phase space for the correlated nucleon pairs in the laboratory system. Color transparency can be studied in $N(d, p p) n$ and $A(d, p p) X$ collisions [69] by the detection of two protons at large transverse momenta. These studies can be complimentary to U-70 and J-PARC scientific programs.

\section{Conclusions}

- The spin structure of $2 \mathrm{~N}$ and $3 \mathrm{~N}$ correlations can be studied at Nuclotron-M both at internal and extracted beams in the few-nucleons interaction.

- The putting into operation new PIS [23] will significantly increase the potentialities of these studies at Nuclotron-M.

- The collider mode and availability of polarized beams provide serious advantages to study $2 \mathrm{~N}$ and $3 \mathrm{~N}$ SRCs at NICA.

The authors would like to express their gratitude to the Nuclotron accelerator staff. We thank Yu.S. Anisimov, A.F. Elishev, Z.P. Kuznezova, J. Kliman, A.P. Laricheva, A.G. Litvinenko, V. Matoucek, M. Morhach, V.G. Perevozchikov, I. Turzo, Yu.V.Zanevsky and V.N. Zhmyrov for the help during the preparation and performance of the experiment. This work was supported in part by the Russian Foundation for Basic Research (Grants $N^{o} 07-02-00102$ a and $N^{o} 10-02-00087 \mathrm{a}$ ), the Grant-in-Aid for Scientific Research (Grant $N^{o} 14740151$ ), by the Grant Agency for Science at the Ministry of Education of the Slovak Republic (Grant $\left.N^{o} 1 / 4010 / 07\right)$ and by a Special program of the Ministry of Education and Science of the Russian Federation(Grant RNP2.1.1.2512). V.P.L. has been supported by RFBR travel Grant $N^{\circ} 09-02-$ $08401 z$.

\section{References}

1. L. Frankfurt, M. Sargsian, and M. Strikman, Int.J.Mod.Phys.A23 (2008) 2991.

2. E. Piasetzky, M. Sargsian, L. Frankfurt, M. Strikman, and J.W. Watson, Phys.Rev.Lett. 97 (2006) 162504. 
3. L.L. Frankfurt, M.I. Strikman, D.B. Day, and M.M. Sargsian, Phys.Rev. C48 (1993) 2451.

4. K.Sh. Egiyan et al., Phys.Rev. C68 (2003) 014313.

5. K.S. Egiyan et al., Phys.Rev.Lett. 96 (2006) 082501.

6. L. Frankfurt, M. Sargsian, and M. Strikman, AIP Conf.Proc.1056 (2008) 322.

7. W. Glöckle, H. Witala, D. Hüber, H. Kamada, and J. Golak, Phys.Rep. 274 (1996) 107.

8. N. Sakamoto et al., Phys.Lett. B367 (1996) 60.

9. K. Sekiguchi et al., Phys.Rev. C65 (2002) 034003.

10. K. Sekiguchi et al., Phys.Rev. C70 (2004) 014001.

11. K. Hatanaka et al., Phys.Rev. C66 (2002) 044002.

12. R. Bieber et al., Phys. Rev. Lett. 84 (2000) 606.

13. K. Ermisch et al., Phys. Rev. Lett. 86 (2001) 5862.

14. K. Ermisch et al., Phys. Rev. C68 (2003) 051001.

15. V.I. Kukulin et al., J. Phys. G: Nucl. Part. Phys. 30 (2004) 287.

16. T. Uesaka et al., Phys.Part.Nucl.Lett 3 (2006) 305.

17. S.M. Piyadin et al., submitted to Eur.Phys.J. ST (2009).

18. A.I. Malakhov et al., Nucl.Instr.Meth. in Phys.Res. A440 (2000) 320.

19. T. Uesaka et al., Phys.Lett. B467 (1999) 199.

20. H. Kamada et al., Prog.Theor.Phys. 104 (2000) 703.

21. T. Uesaka et al., Phys. Lett. B533 (2002) 1.

22. T. Uesaka et al., Nucl.Instr. and Meth. in Phys.Res. A402 (1998) 212.

23. V.V. Fimushkin et al., Eur.Phys.J. ST 162 (2008) 275.

24. K. Suda et al., AIP Conf.Proc. 915 (2007) 920; AIP Conf.Proc. 1011 (2008) 241.

25. P.K. Kurilkin et al., Eur.Phys.J. ST 162 (2008) 137; Int.J.Mod.Phys. A24 (2009) 530.

26. Yu.V. Gurchin et al., Phys.Part.Nucl.Lett. 4 (2007) 263.

27. N.G. Anishchenko et al., AIP Conf.Proc. 95 (1983) 445.

28. K. Suda et al., Nucl.Instr.Meth. in Phys.Res. A572 (2007) 745.

29. H. Witala, private communication.

30. R. Machleidt, Phys. Rev.C 63 (2001) 024001.

31. N.B. Ladygina, Phys.Atom.Nucl. 71 (2008) 2039;

N.B. Ladygina, e-Print: arXiv:0805.3021[nucl-th].

32. M.A. Shikhalev, Phys.Atom.Nucl. 72 (2009) 588.

33. N.B. Ladygina, Eur.Phys.J. A42 (2009) 91.

34. N.E. Booth et al., Phys.Rev. D4 (1971) 1261.

35. J.C. Alder et al., Phys.Rev. C6 (1972) 2010.

36. M. Garson et al., Nucl.Phys. A458 (1986) 287.

37. M. Bleszynski et al., Phys.Lett. B87 (1979) 178; ibid. B106 (1981) 42; M. Haji-Saied et al., Phys.Rev. C36 (1987) 2010.

38. V. Ghazikhanian et al., Phys.Rev. C43 (1987) 1532.

39. V.P. Ladygin et al., Phys.Lett. B629 (2005) 60; L.S. Azhgirey et al., Phys.Atom.Nucl. 71 (2008) 279.

40. A.D. Krisch, Eur.Phys.J. A31 (2007) 423.

41. S. Kistryn et al., Phys.ReV. C68 (2003) 054004; ibid. C72 (2005) 044006.

42. K. Sekiguchi et al., Phys.ReV. C79 (2009) 054008.

43. M. Eslami-Kalantari et al., Mod.Phys.Lett.A24 (2009) 839.

44. E. Stephan et al., Int.J.Mod.Phys.A24 (2009) 515.
45. H. Witala, J. Golak, and R. Skibinski, Phys.Lett.B634 (2006) 374.

46. H. Witala et al., Mod.Phys.Lett.A24 (2009) 871.

47. V.P. Ladygin and S. Nedev, Part. and Nucl.Lett. 2[111]-2002 (2002) 13.

48. V. Punjabi et al., Phys.Lett. B350 (1995) 178.

49. L.S. Azhgirey et al., Phys.Lett. B391 (1997) 22.

50. D. Abbott et al., Phys.Rev.Lett. 84 (2000) 5053.

51. V. Punjabi et al., Phys.Rev. C39 (1989) 608.

52. L.S. Azhgirey et al., Phys.Lett. B387 (1996) 37.

53. A.K. Kurilkin et al., Eur.Phys.J. ST 162 (2008) 133; V.P. Ladygin et al., Phys.Lett. B589 (2004) 47; M. Janek et al., Eur.Phys.J. A33 (2007) 39.

54. N.B. Ladygina, private communication.

55. Y. Shimizu et al., Proc. of the 19-th Few Body Conference (Bonn, Germany, 2009), to be published.

56. M. Janek et al., JINR Preprint P1-2007-171, Dubna (2007).

57. P.A. Rukoyatkin, private communications.

58. A.A. Terekhin et al., to be published in Vestnik BGU (2009).

59. T. Uesaka et al., CNS Report 79, Tokyo (2008).

60. L.S. Azhgirey et al., Instr. and Exp.Tech. 40 (1997) 43; Nucl.Instr.Meth. in Phys.Res. A497 (2003) 340.

61. L.S. Zolin et al., JINR Rapid Comm. 2[88]-98 (1998) 27; Yu.K. Pilipenko et al., AIP Conf. Proc. 570 (2001) 801.

62. Yu.V. Gurchin et al., submitted to Phys.Part.Nucl.Lett. (2009).

63. V.P. Ladygin et al., AIP Conf.Proc. 1056 (2008) 420.

64. L.S. Azhgirey et al., Phys.Part.Nucl.Lett. 4 (2007) 497.

65. V.A. Karmanov and A.V. Smirnov, Nucl.Phys. A546 (1992) 691; ibid. A575 (1994) 520; J. Carbonell and V.A. Karmanov, Nucl.Phys. A581 (1995) 625; ibid. 589 (1995) 713; J. Carbonell, B. Desplanques, V.A. Karmanov, and J.F. Mathiot, Phys.Rep. 300 (1998) 125.

66. S.J. Brodsky and G.R. Farrar, Phys.Rev.Lett. 31 (1973) 1153; Phys.Rev. D11 (1975) 1309; G.P. Lepage and S.J. Brodsky, Phys.Rev. D22 (1980) 2157.

67. V.A. Matveev, R.M. Muradyan, and A.N. Tavkhelidze, Lett. Nuovo Cim. 7 (1973) 719.

68. M. Lacombe, B. Loiseau, R. Vinh Mau, J. Cote, P. Pires, and R. de Tourreil, Phys.Lett. B 101 (1981) 139.

69. L.L. Frankfurt, E. Piasetzky, M.M. Sargsian, and M.I. Strikman, Phys.Rev. C56 (1997) 2752. 